\section{Is Adiponectin New Important Parameter in Adolescents with Metabolic Syndrome?}

\author{
Marijana Tadic ${ }^{1 *}$ and Cesare Cuspidi ${ }^{2}$ \\ ${ }^{1}$ University Clinical Hospital Centre "Dr Dragiša Mišović - Dedinje", School \\ of Medicine, University of Belgrade, Belgrade, Serbia \\ ${ }^{2}$ University of Milan-Bicocca and Istituto Auxologico Italiano, Clinical \\ Research Unit, Meda, Italy
}

\section{Introduction}

Metabolic Syndrome (MS) represents a cluster of cardiovascular risk factors hypertension, obesity, diabetes and dyslipidemia. It has been showed that MS in childhood and adolescence is a significant risk factor for adverse outcomes later in life. Although the obesity prevalence significantly increases in last several decades, the latest studies revealed a linear trend of decreasing MS severity in US and Canadian adolescents $[1,2]$. The decrease in MS severity appeared to be due to trends in increasing High-Density Lipoprotein (HDL) and decreasing triglyceride. The other authors claim that this is the consequence of different MS definitions usage [3].

The central role of the MS is insulin resistance and abdominal obesity. Previous investigations showed unfavorable effect of obesity on cardiac structure, function and mechanics in childhood [4]. This remodeling is related with different hemodynamic and biohumoral changes including activation of sympathetic nervous system and renin-angiotensin system [4]. However, many other biohumoral markers contribute to cardiovascular changes in MS. Recent systematic review revealed that the concentrations of pro-inflammatory cytokines (IL-6, TNF- $\alpha$ ), markers of pro-oxidant status (OxLDL, uric acid), Pro-thrombotic factors (PAI-1) and leptin were elevated, whereas concentrations of anti-inflammatory cytokines (IL-10), adiponectin, ghrelin and antioxidant factors (PON-1) were reduced in subjects with MS [5].

It has been already known that adiponectin, as a protein hormone, modulates a number of metabolic processes, including glucose regulation and fatty acid oxidation. In the recently published study

*Corresponding author: Marijana Tadic, University Clinical Hospital Center "Dr Dragiša Mišović - Dedinje", School of Medicine, University of Belgrade, Heroja Milana Tepića 1, Belgrade, Serbia, Tel: +381 113630600; E-mail: marijana_tadic@hotmail.com

Citation: Tadic M, Cuspidi C (2016) Is Adiponectin New Important Parameter in Adolescents with Metabolic Syndrome? J Diabetes Metab Disord 3: 010.

Received: March 07, 2016; Accepted: March 09, 2016; Published: March 23, 2016

Copyright: (C) 2016 Tadic M and Cuspidi C, This is an open-access article distributed under the terms of the Creative Commons Attribution License, which permits unrestricted use, distribution, and reproduction in any medium, provided the original author and source are credited.
Shafiee et al., investigated the relationship between MS and adiponectin level in 180 adolescents [6]. The authors claimed that adiponectin gradually decreased with the number of MS criteria. Thus, the patients with no MS criteria had the highest value of adiponectin, patients with 1-2 MS criteria had intermediate adiponectin level, whereas MS participants had the lowest adiponectin level, suggesting that the level of insulin resistance gradually increased from subjects without MS criteria to those with at least 3 MS criteria. Importantly, MS was defined according to the adult treatment panel III criteria modified for the pediatric age group. The authors reported significant correlations between adiponectin concentrations and metabolic parameters, except blood pressure. Interestingly, there was no a difference between genders in these correlation analyses. MS remained correlated with adiponectin level in adolescents even after adjustment for age, BMI, total cholesterol and waist circumference [6].

These findings have been previously demonstrated in adult population with MS [7]. However, the results of the latest studies regarding the importance of adiponectin and its relationship with cardiovascular outcome are surprising and unexpected. Namely, one would expect that lower levels of adiponectin are associated with increased glucose intolerance and elevated cardiovascular risk. Nevertheless the newest investigations showed completely opposite situation. The Dallas heart study revealed that higher adiponectin level is associated with increased mortality and cardiovascular morbidity in a young, multiethnic population across all subgroups defined by age, gender, race, obesity, diabetes, MS, or elevated high-sensitivity C-reactive protein. The similar findings were recently detected in hemodialysis and diabetic patients [8-10]. These findings are paradoxical considering the fact that large studies showed that adiponectin inversely correlated with insulin and HOMA index index of insulin resistance [11]. This unexpected direct correlation between high serum adiponectin levels and increased cardiovascular mortality is predominantly based on a cause - effect relationship that potentiates role of adiponectin action and metabolism on atherosclerotic processes. Other study tried to explain the relationship between adiponectin and mortality by the renal function [10]. Namely, the authors found a significant adiponectin-mortality association only in individuals with normal renal function (GFR $\geq 60 \mathrm{ml} / \mathrm{min} / 1.73 \mathrm{~m}^{2}$ ), but not in those with GFR $<60 \mathrm{ml} / \mathrm{min} / 1.73 \mathrm{~m}^{2}$.

The importance of the investigation published by Shafiee et al., lies in the fact that adiponectin level could predict the further development of insulin resistance and diabetes in subjects with MS [6]. The authors confirmed that adiponectin represents a multifunctional protein with pleiotropic insulin-sensitizing effects and a key molecule in the pathogenesis of MS. Additional advantage of this research is the fact that the authors studied Iranian population and showed that the results regarding adiponectin could be applied in multiethnic population. However, further longitudinal studies are needed to confirm the clinical importance of adiponectin assessment and its predictive value in MS subjects. 


\section{References}

1. Lee AM, Gurka MJ, DeBoer MD (2016) Trends in metabolic syndrome severity and lifestyle factors among adolescents. Pediatrics 137: 1-9.

2. MacPherson M, de Groh M, Loukine L, Prud'homme D, Dubois L (2016) Prevalence of metabolic syndrome and its risk factors in Canadian children and adolescents: Canadian Health Measures Survey Cycle 1 (2007-2009) and Cycle 2 (2009-2011). Health Promot Chronic Dis Prev Can 36: 32-40.

3. Asghari G, Eftekharzadeh A, Hosseinpanah F, Ghareh S, Mirmiran P, et al. (2016) Instability of different adolescent metabolic syndrome definitions tracked into early adulthood metabolic syndrome: Tehran Lipid and Glucose Study (TLGS). Pediatr Diabetes.

4. Tadic M, Cuspidi C (2015) Childhood obesity and cardiac remodeling: from cardiac structure to myocardial mechanics. J Cardiovasc Med (Hagerstown) 16: $538-546$.

5. Srikanthan K, Feyh A, Visweshwar H, Shapiro JI, Sodhi K (2016) Systematic review of metabolic syndrome biomarkers: A panel for early detection, management, and risk stratification in the West Virginian population. Int $\mathrm{J}$ Med Sci 13: 25-38.
6. Shafiee G, Ahadi Z, Qorbani M, Kelishadi R, Ziauddin H, et al. (2015) Association of adiponectin and metabolic syndrome in adolescents: the caspian- III study. J Diabetes Metab Disord 14: 89.

7. Vega GL, Grundy SM (2013) Metabolic risk susceptibility in men is partially related to adiponectin/leptin ratio. J Obes 2013: 409679.

8. Rhee CM, Nguyen DV, Moradi H, Brunelli SM, Dukkipati R, et al. (2015) Association of Adiponectin With Body Composition and Mortality in Hemodialysis Patients. Am J Kidney Dis 66: 313-321.

9. Ortega Moreno L, Copetti M, Fontana A, De Bonis C, Salvemini L, et al. (2016) Evidence of a causal relationship between high serum adiponectin levels and increased cardiovascular mortality rate in patients with type 2 diabetes. Cardiovasc Diabetol 15: 17

10. Ortega Moreno L, Lamacchia O, Salvemini L, De Bonis C, De Cosmo S, et al. (2016) The paradoxical association of adiponectin with mortality rate in patients with type 2 diabetes: evidence of synergism with kidney function. Atherosclerosis 245: 222-227.

11. Ding YS, Guo SX, Ma RL, Li SG, Guo H, et al. (2015) Association of Metabolic Syndrome with the Adiponectin to Homeostasis Model Assessment of Insulin Resistance Ratio. Mediators Inflamm 2015: 607364. 